\title{
Using Parallel Monte Carlo Methods in Large-Scale Air Pollution Modelling
}

\author{
V.N. Alexandrov ${ }^{1}$ and Z. Zlatev ${ }^{2}$ \\ 1 Advanced Computing and Emergent Technologies Centre, University of Reading, \\ Reading, UK (v.n.alexandrov@reading.ac.uk) \\ 2 National Environmental Research Institute, Frederiksborgvej 399, P. O. Box 358, \\ DK-4000 Roskilde, Denmark (zz@dmu.dk)
}

\begin{abstract}
Large-scale air pollution models can successfully be used in different environmental studies. These models are described mathematically by systems of partial differential equations. Splitting procedures followed by discretization of the spatial derivatives lead to several large systems of ordinary differential equations of order up to 80 millions. These systems have to be handled numerically at up to 250000 timesteps. Furthermore, many scenarios are often to be run in order to study the dependence of the model results on the variation of some key parameters (as, for example, the emissions). Such huge computational tasks can successfully be treated only if (i) fast and sufficiently accurate numerical methods are used and (ii) the models can efficiently be run on parallel computers. Efficient Monte Carlo methods for some subproblems will be presented and applications of the model in the solution of some environmental tasks will also be made.
\end{abstract}

\section{Introduction}

The control of the pollution levels in different highly polluted regions of Europe and North America (as well as in other highly industrialized parts of the world) is an important task for the modern society. Its relevance has been steadily increasing during the last two-three decades. The need to establish reliable control strategies for the air pollution levels will become even more important in the future. Large-scale air pollution models can successfully be used to design reliable control strategies. Many different tasks have to be solved before starting to run operationally an air pollution model. The following tasks are most important:

- describe in an adequate way all important physical and chemical processes,

- apply fast and sufficiently accurate numerical methods in the different parts of the model,

- ensure that the model runs efficiently on modern high-speed computers (and, first and foremost, on different types of parallel computers),

- use high quality input data (both meteorological data and emission data) in the runs,

- verify the model results by comparing them with reliable measurements taken in different parts of the space domain of the model, 
- carry out some sensitivity experiments to check the response of the model to changes of different key parameters

and

- visualize and animate the output results to make them easily understandable also for non-specialists.

The performance of the model on high-speed computers will be discussed in this paper.

\subsection{Main Physical and Chemical Processes}

Five physical and chemical processes have to be described by mathematical terms in the beginning of the development of an air pollution model. These processes are: (i) horizontal transport (advection), (ii) horizontal diffusion, (iii)chemical transformations in the atmosphere combined with emissions from different sources, (iv) deposition of pollutants to the surface and (v) vertical exchange (containing both vertical transport and vertical diffusion).

It is important to describe in an adequate way all these processes. However, this is an extremely difficult task; both because of the lack of knowledge for some of the processes (this is mainly true for some chemical reactions and for some of the mechanisms describing the vertical diffusion) and because a very rigorous description of some of the processes will lead to huge computational tasks which may make the treatment of the model practically impossible. The main principles used in the mathematical description of the main physical and chemical processes as well as the need to keep the balance between the rigorous description of the processes and the necessity to be able to run the model on the available computers are discussed in [6].

\subsection{Mathematical Formulation of a Large Air Pollution Model}

The description of the physical and chemical processes by mathematical terms leads to a system of partial differential equations (PDEs) of the following type:

$$
\begin{aligned}
\frac{\partial c_{s}}{\partial t}= & -\frac{\partial\left(u c_{s}\right)}{\partial x}-\frac{\partial\left(v c_{s}\right)}{\partial y}-\frac{\partial\left(w c_{s}\right)}{\partial z} \\
& +\frac{\partial}{\partial x}\left(K_{x} \frac{\partial c_{s}}{\partial x}\right)+\frac{\partial}{\partial y}\left(K_{y} \frac{\partial c_{s}}{\partial y}\right)+\frac{\partial}{\partial z}\left(K_{z} \frac{\partial c_{s}}{\partial z}\right) \\
& +E_{s}-\left(\kappa_{1 s}+\kappa_{2 s}\right) c_{s}+Q_{s}\left(c_{1}, c_{2}, \ldots, c_{q}\right), \quad s=1,2, \ldots, q
\end{aligned}
$$

where (i) the concentrations of the chemical species are denoted by $c_{s}$, (ii) $u, v$ and $w$ are wind velocities, (iii) $K_{x}, K_{y}$ and $K_{z}$ are diffusion coefficients, (iv) the emission sources are described by $E_{s},(\mathrm{v}) \kappa_{1 s}$ and $\kappa_{2 s}$ are deposition coefficients 
and (vi) the chemical reactions are denoted by $Q_{s}\left(c_{1}, c_{2}, \ldots, c_{q}\right)$. The CBM IV chemical scheme, which has been proposed in [4], is actually used in the version of DEM (the Danish Eulerian Model; 6], 7]) that will be considered in this paper.

\section{Achieving Parallelism}

Delivering efficient parallel algorithms for treating large scale air pollution is very important. Note, for example, that the size of the computational tasks can be formidable if we need finer resolution, e.g. $480 \times 480$ mesh, leading to solving 8064000 equations per time step and depending on the number of species and time steps potentially to a systems of ordinary differential equations of order up to 80 millions.

Therefore the preparation of a parallel code is by no means an easy task. Moreover, it may happen that when the code is ready the computing centre exchanges the computer which has been used in the preparation of the code with another (hopefully, more powerful) computer. This is why it is desirable to use only standard tools in the preparation of the code. This will facilitate the transition

of the code from one computer to another when this becomes necessary. Only standard MPI ([3]) tools are used in the parallel versions of DEM.

\subsection{Development of MPI Versions of DEM}

The approach used when MPI tools are to be implemented is based in dividing the space domain of the model into $p$ sub-domains, where $p$ is the number of processors which are to be used in the run. Two specific modules are needed in the MPI versions: (i) a pre-processing module and (ii) a post-processing module.

- The pre-processing module. corresponding to the $p$ sub-domains obtained in the division of the space domain. In this way, each processor will work during the whole computational process with its own set of input data.

- The post-processing module. Each processor prepares its own set of output data. During the post-processing the $p$ sets of output data corresponding to the $p$ sub-domains are collected and common output files are prepared for future use.

- Benefits of using the two modules. Excessive communications during the computational process are avoided when the two modules are used. It should be stressed, however, that not all communications during the computational process are avoided. Some communications along the inner boundaries of the sub-domains are still needed. However, these communications are to be carried only once per step and only a few data are to be communicated. Thus, the actual communications that are to be carried out during the computations are rather cheap when the pre-processing and the post-processing modules are proper implemented. 
It is important to emphasize here that the introduction of $p$ sub-domains leads to a reduction of the main arrays by a factor of $p$. Consider as an illustrations the major arrays used in the chemical sub-model. The dimensions of these arrays are reduced from $\left(N_{x} \times N_{y}, N_{s}\right)$ to $\left(N_{x} \times N_{y} / p, N_{s}\right)$. It is clear that this is equivalent to the use of $p$ chunks. Chunks of length $N_{x} \times N_{y} / p$ are still very large. Therefore, the following algorithm has also to be used (in each sub-domain) when the MPI versions are used:

\author{
DO ICHUNK=1,NCHUNKS \\ Copy chunk ICHUNK from some of the eight \\ large arrays into small two-dimensional \\ arrays with leading dimension NSIZE \\ DO $\mathrm{J}=1$,NSPECIES \\ DO $\mathrm{I}=1, \mathrm{NSIZE}$ \\ Perform the chemical reactions involving \\ species $\mathrm{J}$ for grid-point I \\ END DO \\ END DO \\ Copy some of the small two-dimensional \\ arrays with leading dimension NSIZE \\ into chunk ICHUNK of the corresponding \\ large arrays \\ END DO
}

However, the reduction of the arrays leads to a reductions of the copies that are to be made in the beginning and in the end of the algorithm. Thus, the reduction of the arrays leads to a better utilization of the cache memory.

The automatic reduction of the sizes of the involved arrays, and the resulting from this reduction better utilization of the cache memory, make the MPI versions attractive also when shared memory machines are available.

\title{
3 Description of the Grid of Sun Computers
}

Sun computers located at the Danish Centre for Scientific Computing (the Danish Technical University in Lyngby) were used in the runs. The computers and the their characteristics are shown in Table 1. All these computers were connected with a $1 \mathrm{Gbit} / \mathrm{s}$ Switch.

The computers are united in a grid (consisting of 216 processors) so that a job sent without a special demand will be assigned on the computer on which there are sufficiently many free processors. The different computers have processors of different power (therefore, it is in principle possible to use the grid as a heterogeneous architecture, but this option is not available yet).

We are in general allowed to use no more than 16 processors, but several runs on more that 16 processors were performed with a special permission from the Danish Centre for Scientific Computing. In the runs in this section we used only "newton" (i.e. we had always a requirement specifying the particular computer on which the job must be run)

More details about the high speed computers that are available at the Technical University of Denmark can be found in 5]. 
Table 1. The computers available at the Sun grid

\begin{tabular}{|c|l|l|r|c|}
\hline Computer & Type & Power & RAM & Processors \\
\hline Bohr & Sun Fire 6800 & UltraSparc-III 750 MHrz & $48 \mathrm{~GB}$ & 24 \\
\hline Erlang & Sun Fire 6800 & UltraSparc-III 750 MHrz & $48 \mathrm{~GB}$ & 24 \\
\hline Hald & Sun Fire 12k & UltraSparc-III 750 MHrz & $144 \mathrm{~GB}$ & 48 \\
\hline Euler & Sun Fire 6800 & UltraSparc-III 750 MHrz & $24 \mathrm{~GB}$ & 24 \\
\hline Hilbert & Sun Fire 6800 & UltraSparc-III 750 MHrz & $36 \mathrm{~GB}$ & 24 \\
\hline Newton & Sun Fire 15k & UltraSparc-IIIcu 900 MHrz & $404 \mathrm{~GB}$ & 72 \\
\hline
\end{tabular}

\section{Running the MPI Versions of DEM}

Four MPI versions of DEM have been tested: (i) the 2-D model on a coarse grid, (ii) the $3-\mathrm{D}$ version on a coarse grid, (iii) the $2-\mathrm{D}$ version on a fine grid and (iv) the 3 -D version on a fine grid.

The problems were run with three different sizes NSIZE of chunks: (a) the minimal size of the chunks, $N S I Z E=1$ for all cases, (b) a medium size of the chunks, NSIZE $=24$ for all cases and (c) the maximal size of the chunks, which is $N S I Z E=1152$ for the coarse grid when 8 processors are used and $N S I Z E=28800$ for the fine grid (again when 8 processors are used).

Finally, in most of the cases both 1 processor and 8 processors were used. Some of the jobs were also run on more than 8 processors.

All runs of the versions discretized on the coarse grid were run for the typical period of one year (in which case it is possible to study seasonal variations). The 2-D version of DEM discretized on the fine grid was run over a period of one month. Finally, the 3-D version of DEM discretized on the fine grid was run over a time period of 42 hours. This is a rather short period, but it is still meaningful to a certain degree because several changes from day to night and from night to day occur in this period, which is important for the test of the photo-chemical reactions.

The computing times in all tables are given in seconds. The abbreviations used in the tables can be explained as follows:

- ADV stands for the horizontal transport + diffusion process,

- CHEM stands for the process uniting the chemical reactions, the treatment of the emissions and the deposition part,

- COMM stands for the part needed to perform communications along the inner boundaries,

- VERT stands for the vertical exchange processes

- TOTAL stands for the total computing time (including the sum of the times given in the same column above the last item + the computing times needed for performing input-output operations, pre-processing, post-processing, etc.) 
Table 2. Running DEM discretized on a $96 \times 96 \times 10$ grid on one processor

\begin{tabular}{|l|r|r|r|r|r|r|}
\hline & \multicolumn{2}{|c|}{$N S I Z E=1$} & \multicolumn{2}{|c|}{ NSIZE $=24$} & \multicolumn{2}{|c|}{ NSIZE $=1152$} \\
\hline Process & Time & Part & Time & Part & Time & Part \\
\hline ADV & 169776 & $31.5 \%$ & 159450 & $37.8 \%$ & 169865 & $30.9 \%$ \\
\hline CHEM & 337791 & $62.7 \%$ & 233471 & $55.3 \%$ & 348769 & $63.4 \%$ \\
\hline VERT & 23221 & $4.3 \%$ & 21473 & $5.1 \%$ & 23014 & $4.2 \%$ \\
\hline COMM & 2 & $0.0 \%$ & 2 & $0.0 \%$ & 2 & $0.0 \%$ \\
\hline TOTAL & 538953 & $100.0 \%$ & 421763 & $100.0 \%$ & 549835 & $100.0 \%$ \\
\hline
\end{tabular}

Table 3. Running DEM discretized on a $96 \times 96 \times 10$ grid on eight processors

\begin{tabular}{|l|r|r|r|r|r|r|r|r|r|}
\hline & \multicolumn{3}{|c|}{$N S I Z E=1$} & \multicolumn{3}{c|}{$N S I Z E=24$} & \multicolumn{3}{c|}{$N S I Z E=1152$} \\
\hline Process & Time & Part & Speed-up & Time & Part & Speed-up & Time & Part & Speed-up \\
\hline ADV & 18968 & $27.4 \%$ & 9.0 & 18498 & $33.3 \%$ & 8.6 & 18641 & $26.3 \%$ & 9.1 \\
\hline CHEM & 41334 & $59.6 \%$ & 8.2 & 29189 & $52.3 \%$ & 8.0 & 43291 & $61.3 \%$ & 8.1 \\
\hline VERT & 1213 & $1.7 \%$ & 19.1 & 1200 & $2.2 \%$ & 17.9 & 1240 & $1.8 \%$ & 18.6 \\
\hline COMM & 911 & $1.3 \%$ & - & 878 & $1.6 \%$ & - & 973 & $1.4 \%$ & - \\
\hline TOTAL & 69325 & $100.0 \%$ & 7.8 & 55723 & $100.0 \%$ & 7.6 & 70653 & $100.0 \%$ & 7.8 \\
\hline
\end{tabular}

The percentages of the computing times for the different processes related to the total computing times are given in the columns under "Part". The "Speedup" is the ratio of the computing time on one processor and the computing time on $p$ processors (where $p$ is the number of processors that are used in the run under considerations; as mentioned above, eight processors were as a rule used in our experiments).

Running the 3-D MPI version discretized on the coarse grid. Results from the six runs with this code are shown in Table 2 (runs on one processor performed by using three values of $N S I Z E$ ) and Table 3 (runs on 8 processors performed again with three values of $N S I Z E)$.

Running the 3-D MPI version discretized on the fine grid. Results from the six runs with this code are shown in Table 4 (runs on one processor performed by using three values of $N S I Z E$ ) and Table 5 (runs on 8 processors performed again with three values of $N S I Z E)$.

Further, in the next table we present a comparison of the efficiency of using Monte Carlo algorithms [12] for solving subproblems (e.g. linear systems arising after discretization) in the model. Comparisons of the efficiency of these methods with some of the traditional ones such as LU is made. It is seen in Table 6 that with the growth of the problem size Monte Carlo calculations become more efficient than LU for the sparse structured matrices treated in the advection submodel.

Several runs were performed by using up to 60 processors. The $3-\mathrm{D}$ refined version, where high efficiency is most desirable, was used in this runs. The results 
Table 4. Running DEM discretized on a $480 \times 480 \times 10$ grid on one processor

\begin{tabular}{|l|r|r|r|r|r|r|}
\hline & \multicolumn{2}{|c|}{$N S I Z E=1$} & \multicolumn{2}{c|}{$N S I Z E=24$} & \multicolumn{2}{c|}{ NSIZE $=28800$} \\
\hline Process & Time & Part & Time & Part & Time & Part \\
\hline ADV & 261631 & $67.0 \%$ & 271419 & $72.9 \%$ & 268337 & $49.8 \%$ \\
\hline CHEM & 86317 & $22.1 \%$ & 56797 & $15.3 \%$ & 228216 & $42.3 \%$ \\
\hline VERT & 40721 & $10.4 \%$ & 42320 & $11.4 \%$ & 41223 & $7.6 \%$ \\
\hline COMM & 1 & $0.0 \%$ & 1 & $0.0 \%$ & 1 & $0.0 \%$ \\
\hline TOTAL & 390209 & $100.0 \%$ & 372173 & $100.0 \%$ & 539319 & $100.0 \%$ \\
\hline
\end{tabular}

Table 5. Running DEM discretized on a $480 \times 480 \times 10$ grid on eight processors

\begin{tabular}{|l|r|r|r|r|r|r|r|r|r|}
\hline & \multicolumn{3}{|c|}{ NSIZE=1 } & \multicolumn{3}{c|}{ NSIZE $=24$} & \multicolumn{3}{c|}{ NSIZE = 28800 } \\
\hline Process & Time & Part & Speed-up & Time & Part & Speed-up & Time & Part & Speed-up \\
\hline ADV & 13606 & $46.2 \%$ & 19.2 & 13515 & $52.7 \%$ & 20.1 & 13374 & $28.9 \%$ & 20.1 \\
\hline CHEM & 10398 & $35.3 \%$ & 8.3 & 6681 & $26.0 \%$ & 8.5 & 25888 & $56.0 \%$ & 8.8 \\
\hline VERT & 2830 & $9.6 \%$ & 14.4 & 2802 & $10.9 \%$ & 15.1 & 2709 & $5.9 \%$ & 15.2 \\
\hline COMM & 2316 & $7.9 \%$ & - & 2340 & $9.1 \%$ & - & 3925 & $8.5 \%$ & - \\
\hline TOTAL & 29449 & $100.0 \%$ & 13.3 & 25654 & $100.0 \%$ & 14.5 & 46210 & $100.0 \%$ & 11.7 \\
\hline
\end{tabular}

are given in Table 7 and indicate that the parallel algorithms applied in DEM scale very well.

Major conclusions from the runs. It is seen that the exploitation of the cache memory is always giving good results (compare the results for NSIZE = 24 with the results for $N S I Z E=1$ and $N S I Z E=1152(28800)$. The speed-ups for the physical processes are super-linear (greater for ADV and VERT than for CHEM, which should be expected, because chunks are used in the chemical parts). The speed-ups for the total computing time are lower, but anyway at least close to linear.

\section{Conclusion}

It has been shown in this paper, that based on our parallelization approach, different submodels of DEM scale very well when the number of the processors used is increased. For some parts of the model we continue to observe superlinear speedup. In addition, the experiments with Monte Carlo show that for larger problems these algorithms are more efficient that the traditional ones, scale well and can lead to a reduction of computational time.

Further work is required, for example, the improvement of the fine resolution versions of DEM, especially the 3 -D fine resolution version, is an important task which must be resolved in the near future. It is necessary both to improve the performance of the different versions of the model and to have access to more processors (and/or to more powerful computers) in order to be able to run operationally fine resolution versions of DEM. 
Table 6. Running DEM with Monte Carlo in the Advection part for various grid refinements with time step 200, 800 and 2400 respectively

\begin{tabular}{|l|r|r|r|r|r|}
\hline Pr. Size & Method & error & Startup Time & ADVEC & Total Time \\
\hline $8 \times 8$ & $L U$ & 0.001 & 0.0 & 0.090 & 0.090 \\
\hline $8 \times 8$ & $M C$ & 0.001 & 2.1 & 0.090 & 2.190 \\
\hline $32 \times 32$ & $L U$ & 0.001 & 0.01 & 31.06 & 31.07 \\
\hline $32 \times 32$ & $M C$ & 0.001 & 3.52 & 29.230 & 32.72 \\
\hline $96 \times 96$ & $L U$ & 0.001 & 0.01 & 227.57 & 227.58 \\
\hline $96 \times 96$ & $M C$ & 0.001 & 63.8 & 88.8 & 152.6 \\
\hline
\end{tabular}

Table 7. Running DEM discretized on a $480 \times 480 \times 10$ on different numbers of processors

\begin{tabular}{|r|r|r|}
\hline Processors & Time & Speed-up \\
\hline 1 & 372173 & - \\
\hline 15 & 12928 & 28.79 \\
\hline 30 & 7165 & 51.94 \\
\hline 60 & 4081 & 91.20 \\
\hline
\end{tabular}

Acknowledgements. A grant (CPU-1101-17) from the Danish Centre for Scientific Computing (DCSC) gave us access to the Sun computers at the Technical University of Denmark. The members of the staff of DCSC helped us to resolve some difficult problems related to the efficient exploitation of the grid of Sun computers.

\section{References}

1. Alexandrov V.N., Efficient parallel Monte Carlo Methods for Matrix Computation, Mathematics and computers in Simulation, Elsevier 47 pp. 113-122, Netherlands, (1998).

2. Dimov I., Alexandrov V.N. And Karaivanova A., Resolvent Monte Carlo Methods for Linear Algebra Problems, Mathematics and Computers in Simulation, Vo155, pp. 25-36, 2001.

3. W. Gropp, E. Lusk And A. SkJellum, Using MPI: Portable programming with the message passing interface, MIT Press, Cambridge, Massachusetts (1994).

4. M. W. Gery, G. Z. Whitten, J. P. Killus and M. C. Dodge, A photochemical kinetics mechanism for urban and regional computer modeling, Journal of Geophysical Research, Vol. 94 (1989), 12925-12956.

5. WeB-site of the Danish Centre for Scientific Computing at the TechNiCAl University of Denmark, Sun High Performance Computing Systems, http://www.hpc.dtu.dk, 2002.

6. Z. Zlatev, Computer treatment of large air pollution models, Kluwer Academic Publishers, Dordrecht-Boston-London (1995).

7. Z. Zlatev, Massive data set issues in air pollution modelling, In: Handbook on Massive Data Sets (J. Abello, P. M. Pardalos and M. G. C. Resende, eds.), pp. 1169-1220, Kluwer Academic Publishers, Dordrecht-Boston-London (2002). 